\title{
BMJ Open Developing a model to predict individualised treatment for gonorrhoea: a modelling study
}

\author{
Lucy Findlater (D) , ${ }^{1}$ Hamish Mohammed, ${ }^{2}$ Maya Gobin, ${ }^{1}$ Helen Fifer (D) , ${ }^{3}$ \\ Jonathan Ross, ${ }^{4}$ Oliver Geffen Obregon, ${ }^{2}$ Katy M E Turner (i) ${ }^{5}$
}

To cite: Findlater $\mathrm{L}$, Mohammed H, Gobin M, et al. Developing a model to predict individualised treatment for gonorrhoea: a modelling study. BMJ Open 2021;11:e042893. doi:10.1136/ bmjopen-2020-042893

- Prepublication history and supplemental material for this paper is available online. To view these files, please visit the journal online (http://dx.doi. org/10.1136/bmjopen-2020042893).

Received 22 July 2020 Accepted 29 April 2021

Check for updates

C Author(s) (or their employer(s)) 2021. Re-use permitted under CC BY-NC. No commercial re-use. See rights and permissions. Published by BMJ.

${ }^{1}$ National Infection Service, Public Health England, Bristol, UK

${ }^{2}$ HIV \& STI Department, Public Health England, London, UK

${ }^{3}$ Reference Microbiology, Public Health England, London, UK ${ }^{4}$ Institute of Microbiology and Infection, University Hospitals Birmingham NHS Foundation

Trust, Birmingham, UK

${ }^{5}$ Bristol Veterinary School, University of Bristol, Bristol, UK

Correspondence to

Lucy Findlater;

lucy.findlater@phe.gov.uk

\section{ABSTRACT}

Objective To develop a tool predicting individualised treatment for gonorrhoea, enabling treatment with previously recommended antibiotics, to reduce use of lastline treatment ceftriaxone.

Design A modelling study.

Setting England and Wales.

Participants Individuals accessing sentinel health services.

Intervention Developing an Excel model which uses participants' demographic, behavioural and clinical characteristics to predict susceptibility to legacy antibiotics. Model parameters were calculated using data for 2015-2017 from the Gonococcal Resistance to Antimicrobials Surveillance Programme.

Main outcome measures Estimated number of doses of ceftriaxone saved, and number of people delayed effective treatment, by model use in clinical practice. Model outputs are the predicted risk of resistance to ciprofloxacin, azithromycin, penicillin and cefixime, in groups of individuals with different combinations of characteristics (gender, sexual orientation, number of recent sexual partners, age, ethnicity), and a treatment recommendation.

Results Between 2015 and 2017, 8013 isolates were collected: $64 \%$ from men who have sex with men, $18 \%$ from heterosexual men and 18\% from women. Across participant subgroups, stratified by all predictors, resistance prevalence was high for ciprofloxacin (range: $11 \%-51 \%$ ) and penicillin (range: 6\%-33\%). Resistance prevalence for azithromycin and cefixime ranged from $0 \%$ to $13 \%$ and for ceftriaxone it was $0 \%$. Simulating model use, $88 \%$ of individuals could be given cefixime and $10 \%$ azithromycin, saving $97 \%$ of ceftriaxone doses, with $1 \%$ of individuals delayed effective treatment.

Conclusions Using demographic and behavioural characteristics, we could not reliably identify a participant subset in which ciprofloxacin or penicillin would be effective. Cefixime resistance was almost universally low; however, substituting ceftriaxone for near-uniform treatment with cefixime risks reemergence of resistance to cefixime and ceftriaxone. Several subgroups had low azithromycin resistance, but widespread azithromycin monotherapy risks resistance at population level. However, this dataset had limitations; further exploration of individual characteristics to predict resistance to a wider range of legacy antibiotics may still be appropriate.

\section{Strengths and limitations of this study}

- Model predicts individual susceptibility to legacy antibiotics for gonorrhoea and provides treatment recommendation.

- Estimates number of doses of last-line treatment ceftriaxone saved if model used in clinical practice.

- Uses contemporary surveillance data on antibiotic resistance.

- Simple, user-friendly Excel model could be updated with more recent surveillance data or additional risk factors.

- Small sample sizes limited the choice and number of risk factors in the model and the reliability of prevalence estimates used.

\section{INTRODUCTION}

Gonorrhoea is the second most common bacterial sexually transmitted infection (STI) diagnosed in the UK. In 2018, 56259 diagnoses were reported in England, which is $26 \%$ more than in $2017 .^{12}$ If untreated, gonorrhoea can lead to urethritis, cervicitis, pelvic inflammatory disease and infertility. ${ }^{34}$ Neisseria gonorrhoeae has developed resistance to all antibiotics previously used to treat it. ${ }^{35}$ From sulfonamides, penicillin, tetracycline, azithromycin and ciprofloxacin to cefixime, the bacterium has become resistant to each new treatment in turn, including, in rare cases, to current and last-line treatment ceftriaxone. $^{67}$ In the UK, individuals diagnosed with gonorrhoea are prescribed $1 \mathrm{~g}$ of ceftriaxone administered by intramuscular injection; in other countries, dual therapy of ceftriaxone (250-500 mg) with oral azithromycin (1-2 g) is recommended. ${ }^{8-10}$

The Gonococcal Resistance to Antimicrobials Surveillance Programme (GRASP), a sentinel surveillance scheme, has been monitoring antimicrobial resistance (AMR) in $N$. gonorrhoeae in England and Wales since 2000 (figure 1). ${ }^{811}$ Several cases of ceftriaxoneresistant gonorrhoea have been reported as 


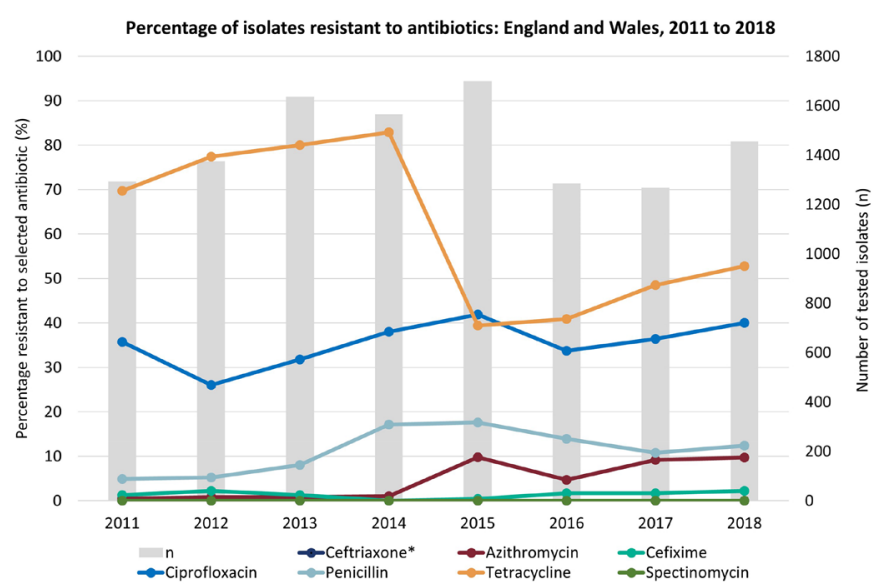

Figure 1 Trends in gonococcal antibiotic resistance, 2011-2018. Data extracted from Public Health England. ${ }^{8}$ The percentage of isolates resistant to ceftriaxone was $0 \%$ from 2011 to 2018.

well as an increase in the proportion of isolates with a higher minimum inhibitory concentration (MIC) for ceftriaxone, indicating a reduction in sensitivity to the antibiotic. over time, this upwards drift in MIC is likely to result in ceftriaxone resistance. ${ }^{812} 13$

Given the reduction in sensitivity to ceftriaxone and multiple reports of treatment failure, alternative treatment strategies should be considered to prepare for the emerging threat of ceftriaxone resistance. ${ }^{812}$ This could involve treating cases with previously recommended antibiotics to prolong the lifetime of ceftriaxone as an effective treatment. Current therapy with ceftriaxone tends to be based empirically rather than on individual isolate susceptibility, often because no culture result is available at the time of treatment; this approach assumes all infections are resistant to previous treatments, which in most cases is untrue. ${ }^{2}$ In addition, resistance prevalence for some legacy antibiotics has decreased as their usage has decreased, for example, for cefixime. The prevalence of AMR is also likely to vary in different subgroups of individuals. For example, antibiotic-susceptible strains are thought to be circulating in low-risk groups, such as those who are less likely to be exposed to antibiotics. ${ }^{14}$ This means many people who are currently treated with last-line therapy could potentially be treated with legacy antibiotics. $^{714}$

This study aimed to develop an Excel model predicting susceptibility to legacy antibiotics, based on individual demographic, behavioural and clinical characteristics. This could enable individualised treatment of gonorrhoea with legacy antibiotics for people with a very low estimated risk of resistance. GRASP data were used to estimate the prevalence of AMR in different groups of individuals, attempting to identify groups with a very low prevalence of AMR through an exploratory approach. These estimates were fed into the model, which provides the individual's risk of AMR and a recommended treatment option. Using the tool in clinical practice to guide treatment could reduce the number of doses of ceftriaxone given and preserve its use for strains requiring last-line therapy. This could prolong the lifetime of ceftriaxone as an effective antibiotic by reducing selection pressure and delaying the emergence of resistance.

\section{METHODS}

\section{Data sources}

The study used pseudonymised, individual-level data from the GRASP surveillance dataset, between 2015 and 2017. The GRASP programme involves collecting clinical isolates from consecutive patients diagnosed with gonorrhoea each year between July and September, from sentinel sexual health clinics in England and Wales. The isolates are tested for antimicrobial susceptibility and MICs are recorded. The data are matched to demographic, clinical and behavioural characteristics submitted by the clinics. ${ }^{12} 15$ The Diagnostic Sensitivity Test agar, the medium used by GRASP for susceptibility testing of isolates, was changed in 2015; this affected the MICs for azithromycin and tetracycline. ${ }^{16} 17$ To avoid difficulties interpreting data from across this period, we used data from 2015 onwards.

\section{Data analysis}

We explored the association between participant characteristics and AMR to decide which risk factors to include in the model. GRASP data extraction and analyses were conducted in STATA V.13. This study used the MIC thresholds chosen by GRASP to define gonococcal resistance, which align with the breakpoints used by the European Committee on Antimicrobial Susceptibility (EUCAST) in 2018. (table 1$)^{1618}$ It is worth noting that the EUCAST breakpoint for azithromycin has since been replaced with an epidemiological cut-off value of $1.0 \mathrm{mg} / \mathrm{L}$, but the previous breakpoint of $0.5 \mathrm{mg} / \mathrm{L}$ has been retained in GRASP reports for continuity. ${ }^{19}{ }^{20}$ We investigated risk factors which have been shown or are suspected to be associated with reduced antimicrobial susceptibility: for example, men who have sex with men (MSM) have been found to have higher rates of AMR than heterosexual men, bearing in mind that there may not always be truthful disclosure of sexual orientation in healthcare settings. ${ }^{142122}$ Other characteristics such as age and gender have also been proposed as a risk factors associated with

\begin{tabular}{ll}
\hline $\begin{array}{l}\text {l} ~ \\
\text { Table } 1 \text { Breakpoints used to define antimicrobial } \\
\text { resistance }\end{array}$ \\
\hline Antimicrobial & Resistance definition (MIC) \\
\hline Ceftriaxone & $>0.125 \mathrm{mg} / \mathrm{L}$ \\
Azithromycin & $>0.5 \mathrm{mg} / \mathrm{L}$ \\
Cefixime & $>0.125 \mathrm{mg} / \mathrm{L}$ \\
Ciprofloxacin & $>0.06 \mathrm{mg} / \mathrm{L}$ \\
Penicillin & $>1 \mathrm{mg} / \mathrm{L}$ \\
\hline
\end{tabular}

MIC, minimum inhibitory concentration. 
resistance. ${ }^{14}$ Overall, we investigated the characteristics: gender, sexual orientation, age, ethnicity and number of recent sexual partners (within the previous 3 months), as well as HIV status, previous gonorrhoea and concurrent STIs, and their correlation with each other. We conducted logistic regression to determine ORs for the associations between antibiotic resistance and participant characteristics. Stratified analysis was conducted to obtain estimates of the prevalence of resistance in subgroups of individuals with different combinations of the characteristics found to be associated with resistance.

We chose to investigate resistance to the antibiotics ciprofloxacin, azithromycin, penicillin and cefixime, as they have all been previously recommended to treat gonorrhoea, and resistance to each is well described in the GRASP dataset. Although azithromycin is used alongside ceftriaxone as first-line therapy worldwide, this is not currently recommended in the UK; therefore, we classed azithromycin as a legacy antibiotic.

\section{Model}

We developed a user-friendly model in Microsoft Excel (2016 version) to predict individualised treatment for gonorrhoea. It consists of an input table to enter individual characteristics; a lookup table containing estimates of the prevalence of resistance derived from GRASP data; and an output table providing the risk of resistance to multiple antibiotics and a recommended treatment. We developed the treatment recommendation algorithm based on the WHO guidelines. ${ }^{23}$

\section{Simulation}

We estimated the impact of using the tool in clinical practice in a hypothetical population, estimating the number of doses of ceftriaxone saved and number of people delayed effective treatment. We incorporated the predicted outcome at test-of-cure (TOC) at the 2-week follow-up consultation recommended in BASHH treatment guidelines. ${ }^{4}$ We assumed that the treatment failure rate was equal to the estimated risk of resistance for that subgroup, and that if treatment was found to be unsuccessful after the TOC, the individual would then be prescribed ceftriaxone. We estimated the total number of doses of ceftriaxone saved by calculating the doses saved initially by prescribing legacy antibiotics instead of ceftriaxone, minus doses of ceftriaxone given at follow-up if treatment was unsuccessful. Other assumptions were that treatment failure was only due to resistance, rather than other factors such as non-adherence; there was no re-infection; and all individuals attended follow-up.

We simulated use of the model at the $5 \%$ threshold as the highest acceptable risk of resistance in a legacy antibiotic for it to be prescribed, as well as at the $10 \%$ and $20 \%$ thresholds as a sensitivity analysis to explore a less cautious approach. We also repeated the simulation using the upper and lower bounds of the CIs for the estimates of the risk of resistance in each subgroup, allowing us to investigate the impact of a higher or lower prevalence of resistance than predicted. For groups in which the prevalence of resistance was estimated to be zero, the lower bound was taken to be 0 , and a value of 0.25 was selected as the upper bound, based on a similar approach used in other studies. ${ }^{24}$

\section{Patient and public involvement}

Patients or the public were not involved in the design, or conduct, or reporting, or dissemination plans of this research.

\section{RESULTS}

\section{Sample characteristics}

Between 2015 and 2017, 8013 isolates were collected by GRASP, along with characteristics such as gender and sexual orientation, and tested for antimicrobial susceptibility. Isolates were collected from 29 free at the point of use, specialist sexual health clinics across England and Wales. ${ }^{8}$ The average number of samples submitted by each clinic was 276, ranging from 18 to 1567 samples per clinic. In total, $63.5 \%$ of isolates were from MSM, $18.4 \%$ were from heterosexual men and $18.1 \%$ were from heterosexual women. Overall, $39.5 \%$ of isolates were resistant to ciprofloxacin; $8.2 \%$ to azithromycin; $14.1 \%$ to penicillin; and $1.2 \%$ to cefixime. Data cleaning was conducted prior to analysis: for the 8494 recorded observations, there were 8014 attendances where both patient information and isolates were collected; removing a duplicated observation left 8013 observations remaining. Clinic re-attendance was explored during analysis, identified by duplication of clinic-specific patient ID codes with different accompanying information, for example dates of visit. A total of 8013 clinic attendances occurred in 7765 individuals, due to re-attendance. In total, 224 people attended the same clinic twice $(2.9 \%) ; 12$ people attended the same clinic three times $(0.2 \%)$. The remaining 7529 people did not re-attend $(97.0 \%)$. Re-attendances were not removed from analysis but retained, acting as separate treatment episodes. Cases where the same people attended different clinics could not be identified as patient ID codes were clinic specific.

\section{Relationships between risk factors and antibiotic resistance}

We explored associations between individual characteristics and AMR to determine which variables should be included in the model, using GRASP surveillance data from 2015 to 2017. We combined gender and sexual orientation into a single variable grouped into women, heterosexual men and MSM; this was due to very low numbers of women identifying as bisexual or homosexual. We could not conduct logistic regression for certain variables, for example, concurrent STIs, due to missing data.

The odds of AMR, calculated with univariate ORs, were greater in men than women for all antibiotics, and greater in MSM than heterosexual men for all antibiotics except cefixime, for which MSM had significantly reduced odds of resistance (OR $0.24,95 \%$ CI $0.11-0.50, \mathrm{p}=0.000$ ) 
(table 2). The odds of AMR were greater in older than younger individuals for all antibiotics except cefixime, where older individuals had lower odds. The same pattern was observed for ethnicity: white individuals had higher odds of AMR for all antibiotics except cefixime. Having more than two recent sexual partners was associated with greater odds of ciprofloxacin resistance and reduced odds of resistance to azithromycin, penicillin or cefixime.

\section{Subgroup-specific analysis}

We conducted stratified analysis to estimate the prevalence of resistance in participant subgroups based on combinations of risk factors associated with AMR: age, number of sexual partners in the previous 3 months, gender, sexual orientation and ethnicity (figure 2 ). These variables were chosen because of their association with higher or lower prevalence of AMR. We also described the correlation of these variables with each other (online supplemental table A1). High levels of missing data for variables such as HIV status and previous gonorrhoea infection prohibited their inclusion in stratified analysis. ${ }^{12}$ Notably, the variable for the site of infection (eg, urethral, cervical or pharyngeal) was not included in the model as it was missing or unknown for $41 \%$ of observations in the dataset; the variable for geographic region was also excluded as it was missing for $64 \%$ of observations (online supplemental tables A2 and A3). Some variables were converted to binary variables for the stratified analysis, as including more categories led to low numbers of individuals per stratum. The cut-off points chosen for binarisation were aimed to be discriminatory, resulting in a large difference in the prevalence of AMR between groups, while remaining clinically relevant. Therefore, the cut-offs chosen were as follows: number of partners $(<2$ or $\geq 2$ partners), age ( $<25$ or $\geq 25$ years), ethnicity (white or other). Importantly, missing data and low numbers of individuals per stratum limited the choice and number of variables included in the stratified analysis. Of 8494 total sample observations, 4944 were included in the stratified analysis $(58.2 \%)$.

Estimates of the prevalence of resistance were obtained for 24 different participant groups. For ciprofloxacin, resistance was high in all groups, with a median of $31.6 \%$, varying from $11.1 \%$ to $54.1 \%$; resistance was lowest in women with at least two recent sexual partners, at least 25 years old and of non-white ethnicity (table 3 , figure 2 ). For azithromycin, the median resistance prevalence was $6.2 \%$, with a maximum value of $12.7 \%$. Five groups $(6.3 \%$ of participants) had no recorded azithromycin resistance, while multiple groups had a prevalence of resistance less than $5 \%$, the WHO treatment threshold (which made up $18.3 \%$ of participants included in the model). ${ }^{9}$ For penicillin, resistance was relatively high, with a median of $13.5 \%$; this varied from $5.8 \%$ to $33.3 \%$ and never fell below the $5 \%$ recommended WHO limit, and was lowest in women with at least two recent sexual partners, younger than 25 and of white ethnicity. For cefixime, the median resistance prevalence was $1.9 \%$; nine subgroups had no recorded resistance (making up 33.7\% of participants). The highest level of cefixime resistance recorded was 13.3\%; otherwise, the estimated prevalence of cefixime resistance was almost universally low. In total, $96.3 \%$ of participants fell into groups with an estimated prevalence of cefixime resistance below $5 \%$.

\section{Developing the model}

We developed the model as an Excel spreadsheet allowing the input of individual characteristics and providing an output of their predicted risk of resistance to ciprofloxacin, azithromycin, penicillin and cefixime and a treatment recommendation of the lowest-risk antibiotic. The model (Model, online supplemental file) and user guide (Model-user guide, online supplemental file 1) are available as additional files. We developed the recommended treatment algorithm such that if the estimated risk of resistance to an older antibiotic is $5 \%$ or less, this antibiotic will be recommended instead of ceftriaxone. This is based on WHO guidelines specifying that a treatment should only be recommended if the chance of success is at least $95 \% .{ }^{23}$ The threshold used in the model is modifiable to suit the user's judgement.

\section{Simulation of the impact of the model}

We simulated the impact of the model if used in routine clinical practice with a hypothetical population of 50000 individuals diagnosed with gonorrhoea, based on the PHE annual STI data tables for 2018, assumed to have the same distribution of characteristics as the sample included in stratified analysis. ${ }^{1}$ We estimated that, using the tool at the $5 \%$ resistance threshold for recommending an antibiotic, $98.5 \%$ of people (49 252) attending GUM clinics with gonorrhoea could be treated initially with cefixime or azithromycin instead of ceftriaxone (table 4). The prevalence of ciprofloxacin and penicillin resistance was too high for any groups to be treated with these agents. Only heterosexual men aged under 25 years with fewer than two recent sexual partners, of white ethnicity, would be initially given ceftriaxone. Treatment would be unsuccessful for $1.0 \%$ of people (519), who would then be prescribed ceftriaxone at follow-up, meaning the cure rate with the model is estimated at $99.0 \%$. This leads to an overall value of $97.47 \%$ of doses of ceftriaxone saved (48 733). We repeated this analysis at the $10 \%$ and $20 \%$ thresholds for antibiotic recommendation to estimate the impact of less cautious approach to prescribing legacy antibiotics; this could save more doses of ceftriaxone, but risks more people being delayed effective treatment due to resistance. At the $10 \%$ and $20 \%$ levels, all individuals were initially treated with cefixime or azithromycin, as the risk of resistance to these antibiotics was always estimated to be lower than resistance to ciprofloxacin and penicillin. Overall, 98.8\% of doses of ceftriaxone (49 422) were saved, while $1.2 \%$ of people (578) were delayed effective treatment (cure rate 98.8\%). We also repeated the simulation using the upper and lower bounds of the CIs for the estimates of resistance prevalence (table 4), 


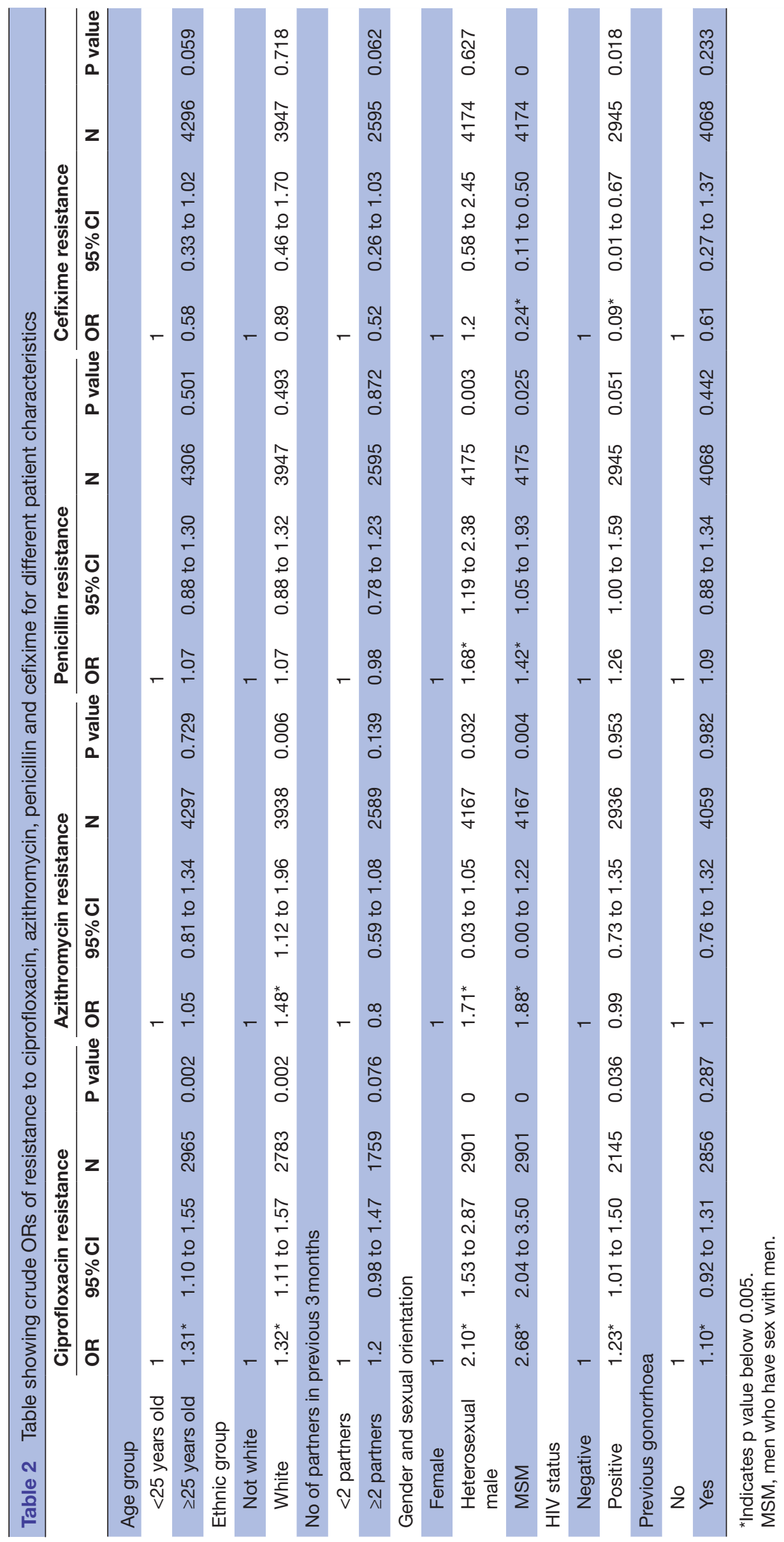



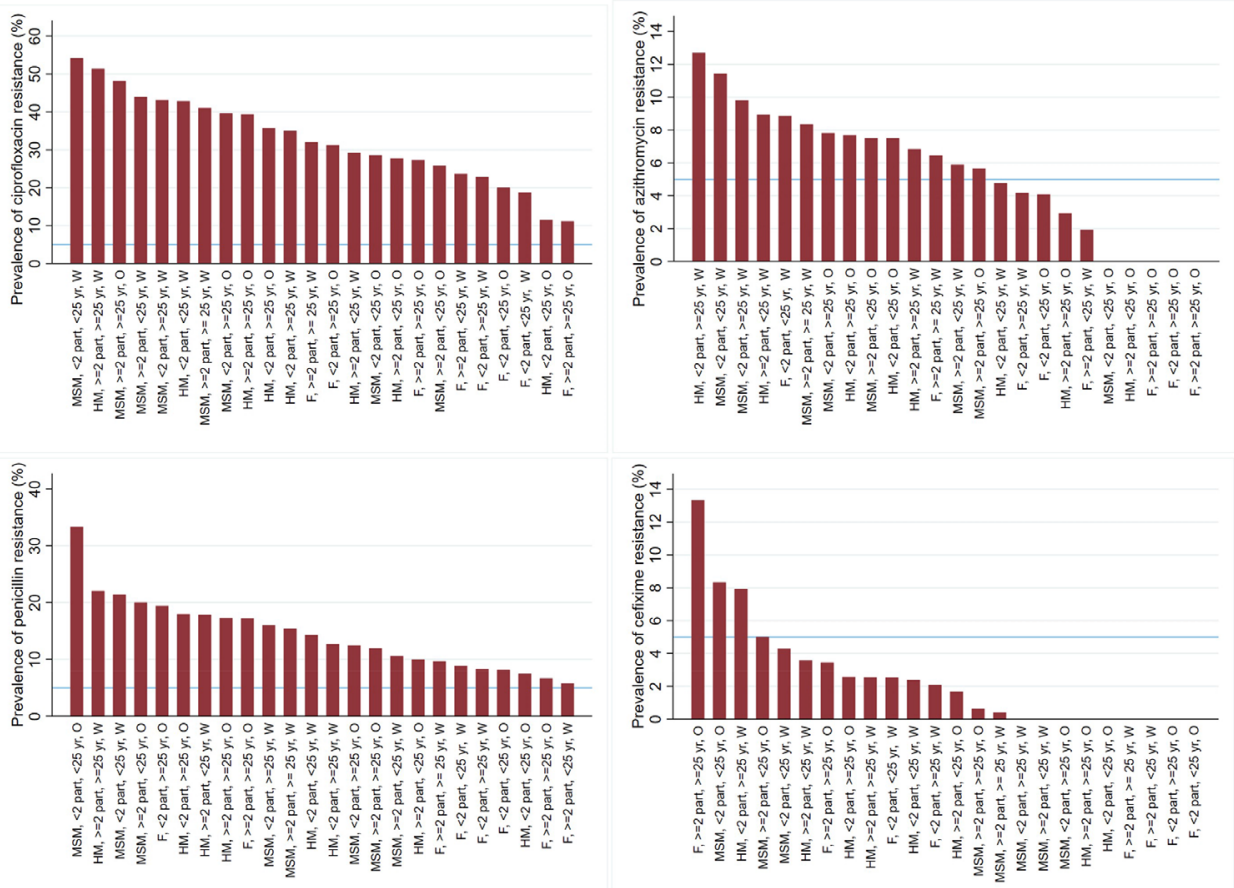

Figure 2 Graphs showing the variation in the prevalence of antibiotic resistance in different patient subgroups. The blue line represents the $5 \%$ resistance threshold, below which the antibiotic can be recommended. HM, heterosexual male; MSM, men who have sex with men.

investigating the impact of uncertainty in the prevalence estimates. Using the lower bound (assuming resistance prevalence is lower than predicted), with $5 \%, 10 \%$ or $20 \%$ treatment thresholds, $99.7 \%$ (49 844) of doses of ceftriaxone were saved. Using the upper bound, with a $5 \%$ threshold, $73.4 \%(36,700)$ doses of ceftriaxone were saved. This rose to $83.9 \%$ at the $10 \%$ level and $96.2 \%$ at the $20 \%$ level.

\section{DISCUSSION}

\section{Statement of principal findings}

In this study, we developed a model predicting individual susceptibility to antibiotics based on patient characteristics associated with resistance: gender, sexual orientation, age, ethnicity and number of recent sexual partners. This was achieved by calculating estimates of the prevalence of resistance in different participant subgroups, using GRASP data. We estimated that resistance to ciprofloxacin and penicillin was too high for treatment in all groups, never dropping below $11.1 \%$ for ciprofloxacin or $5.8 \%$ for penicillin. ${ }^{23}$ For cefixime and azithromycin, the estimated prevalence of resistance was lower, estimated to be below the WHO $5 \%$ treatment recommendation threshold for patients groups making up $96.3 \%$ of individuals (cefixime) or $18.3 \%$ (azithromycin).

The model could be a novel method of rapidly establishing individual susceptibility to a range of antibiotics, by using population-level data to predict individual susceptibility. However, use of the tool in practice would depend on the prevalence of resistance being sufficiently varied between groups to enable heterogenous antibiotic prescription. If AMR was predicted to be universally low and all participants were prescribed one legacy antibiotic, resistance could rapidly re-emerge. If AMR was universally high, no legacy antibiotics would be prescribed, and the situation would be unchanged. Ideally, a minority of small groups of individuals could be safely treated with a range of different legacy antibiotics without prompting the rapid return of resistance to any one antibiotic. When we simulated routine use of the model in clinical practice, we estimated that $97.5 \%$ of doses of ceftriaxone could be saved by treating people almost universally with cefixime (88.2\% of participants) or azithromycin (10.3\%). However, substituting ceftriaxone for cefixime in the majority of patients would be undesirable as it could prompt the return of widespread cefixime resistance and potentially also select for ceftriaxone resistance, while widespread use of azithromycin monotherapy also risks the return of azithromycin resistance. ${ }^{25}$ Therefore, the analysis suggests that there may not be enough variation in patient susceptibility to these antibiotics for this approach to be feasible in the current context.

\section{Strengths and weaknesses of the study}

We derived model parameters from contemporary data on antibiotic resistance and individual characteristics in England and Wales. The GRASP dataset is considered to have robust methodology for determining AMR and provides a valuable resource in combining resistance data with a variety of individual characteristics. ${ }^{12} 15$ This study uses a simple, user-friendly Excel tool to predict treatment. The tool could be modified to be updated with more recent resistance data, different risk factors 


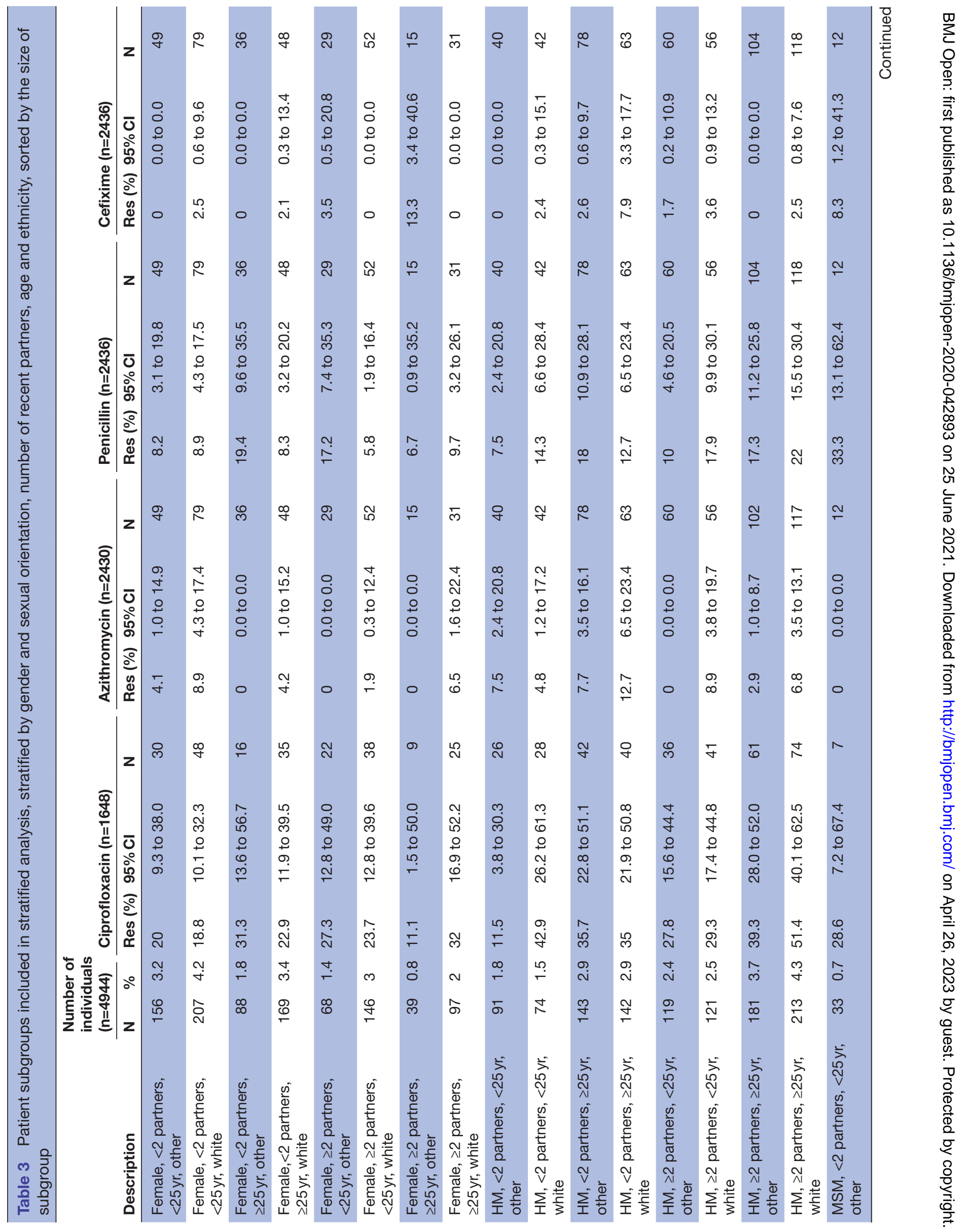




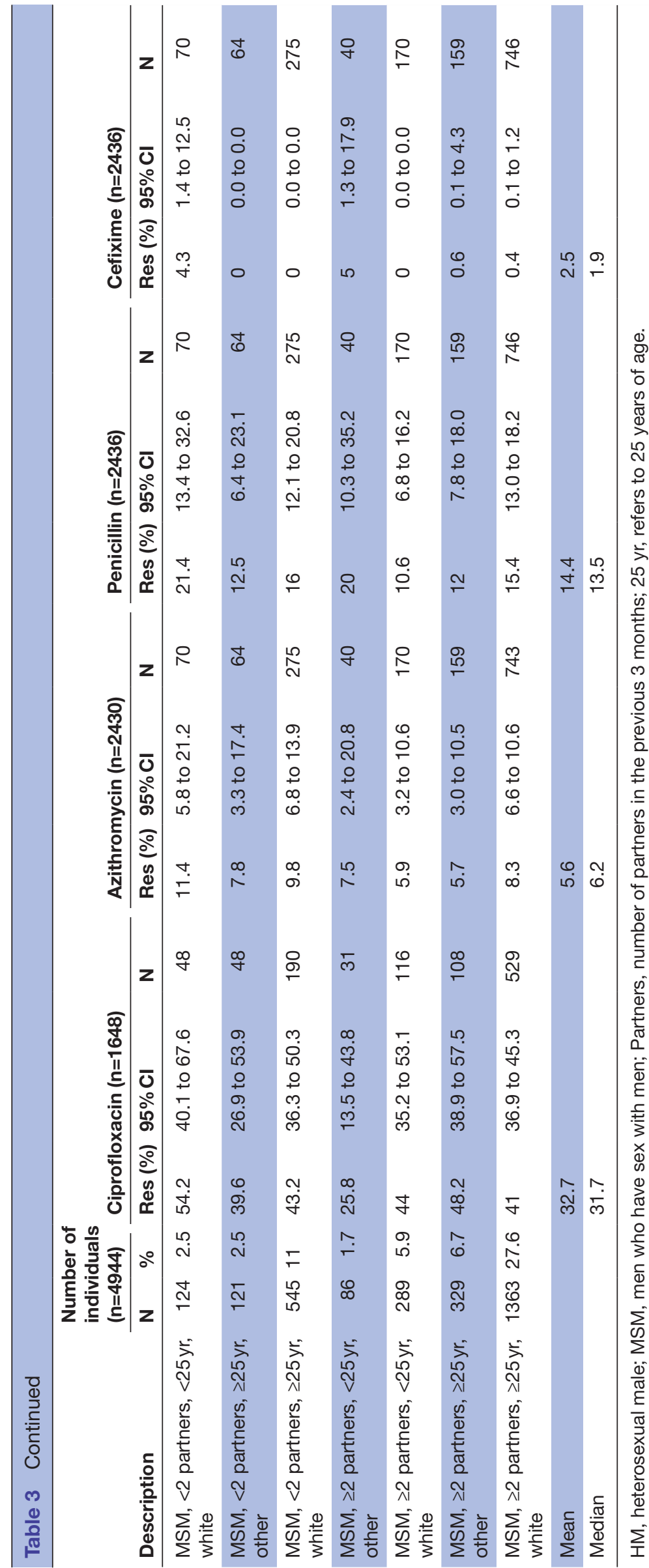

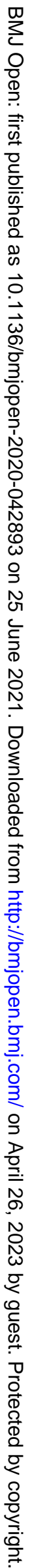


Table 4 Simulation of the impact of using the Excel tool in practice, using the baseline, lower bound and upper bound of the Cls for the estimated prevalence of resistance in each patient subgroup

\begin{tabular}{|c|c|c|c|c|c|c|c|}
\hline & & \multicolumn{2}{|c|}{ Initial treatment } & \multicolumn{2}{|c|}{ Follow-up } & \multicolumn{2}{|l|}{ Overall } \\
\hline & & $\mathbf{N}$ & $\%$ & $\mathbf{N}$ & $\%$ & $\mathbf{N}$ & $\%$ \\
\hline \multicolumn{8}{|l|}{ Baseline } \\
\hline \multirow[t]{4}{*}{$5 \%$ Level } & Ceftriaxone given & 748 & 1.5 & 519 & 1.04 & 1267 & 2.53 \\
\hline & Doses saved & 49252 & 98.5 & 49481 & 98.96 & 48733 & 97.47 \\
\hline & Cefixime & 44094 & 88.19 & & & & \\
\hline & Azithromycin & 5158 & 10.52 & & & & \\
\hline \multirow[t]{4}{*}{ 10\% Level } & Ceftriaxone given & 0 & 0 & 578 & 1.16 & 578 & 1.16 \\
\hline & Doses saved & 50000 & 100 & 49422 & 98.84 & 49422 & 98.84 \\
\hline & Cefixime & 44842 & 89.68 & & & & \\
\hline & Azithromycin & 5158 & 10.32 & & & & \\
\hline \multirow[t]{4}{*}{ 20\% Level } & Ceftriaxone given & 0 & 0 & 578 & 1.16 & 578 & 1.16 \\
\hline & Doses saved & 50000 & 100 & 49422 & 98.84 & 49422 & 98.84 \\
\hline & Cefixime & 44842 & 89.68 & & & & \\
\hline & Azithromycin & 5158 & 10.32 & & & & \\
\hline \multicolumn{8}{|c|}{ Lower bound of $\mathrm{Cl}$} \\
\hline \multirow[t]{4}{*}{ 5\% Level } & Ceftriaxone given & 0 & 0 & 156 & 0.31 & 156 & 0.31 \\
\hline & Doses saved & 50000 & 100 & 49844 & 99.69 & 49844 & 99.69 \\
\hline & Cefixime & 46491 & 92.98 & & & & \\
\hline & Azithromycin & 3509 & 7.02 & & & & \\
\hline \multirow[t]{4}{*}{$10 \%$ Level } & Ceftriaxone given & 0 & 0 & 156 & 0.31 & 156 & 0.31 \\
\hline & Doses saved & 50000 & 100 & 49844 & 99.69 & 49844 & 99.69 \\
\hline & Cefixime & 46491 & 92.98 & & & & \\
\hline & Azithromycin & 3509 & 7.02 & & & & \\
\hline \multirow[t]{4}{*}{ 20\% Level } & Ceftriaxone given & 0 & 0 & 156 & 0.31 & 156 & 0.31 \\
\hline & Doses saved & 50000 & 100 & 49844 & 99.69 & 49844 & 99.69 \\
\hline & Cefixime & 46491 & 92.98 & & & & \\
\hline & Azithromycin & 3509 & 7.02 & & & & \\
\hline \multicolumn{8}{|c|}{ Upper bound of $\mathrm{Cl}$} \\
\hline \multirow[t]{4}{*}{$5 \%$ Level } & Ceftriaxone given & 12935 & 25.87 & 365 & 0.73 & 13300 & 26.6 \\
\hline & Doses saved & 37065 & 74.13 & 49635 & 99.27 & 36700 & 73.4 \\
\hline & Cefixime & 33556 & 67.11 & & & & \\
\hline & Azithromycin & 3509 & 7.02 & & & & \\
\hline \multirow[t]{4}{*}{$10 \%$ Level } & Ceftriaxone given & 7241 & 14.48 & 819 & 1.64 & 8060 & 16.12 \\
\hline & Doses saved & 42759 & 85.52 & 49181 & 98.36 & 41940 & 83.88 \\
\hline & Cefixime & 39250 & 78.5 & & & & \\
\hline & Azithromycin & 3509 & 7.02 & & & & \\
\hline \multirow[t]{4}{*}{ 20\% Level } & Ceftriaxone given & 0 & 0 & 1888 & 3.78 & 1888 & 3.78 \\
\hline & Doses saved & 50000 & 100 & 48112 & 96.22 & 48112 & 96.22 \\
\hline & Cefixime & 46491 & 92.98 & & & & \\
\hline & Azithromycin & 3509 & 7.02 & & & & \\
\hline
\end{tabular}

Follow-up, follow-up consultation 2 weeks after initial treatment.

or other antibiotics. The ability to modify the resistance threshold for prescribing an antibiotic makes the tool more versatile and acceptable. This simple approach to stratifying treatment based on individual characteristics is transferable and could be applied to the management of other infectious diseases, for example urinary 
tract infections, provided that high-quality, reliable data are available on the trends of AMR among that population. ${ }^{526}$ More broadly, an individualised approach to antibiotic prescribing could lead to increased heterogeneity of treatments given, lessening the selection pressure on the emergence of resistance to last-line therapy. ${ }^{6}$

Oversampling of some groups in the GRASP dataset may be associated with differences in the prevalence of resistance between participant groups. For example, MSM are more likely to present for treatment at sentinel sites and are a higher-risk group for AMR; this could lead to overestimating the prevalence of resistance in our sample. ${ }^{15}$ In addition, small sample sizes were used to obtain subgroup-specific resistance estimates. This is partly because the GRASP dataset was restricted to 3 years of data (2015-2017) due to a change in the medium used for susceptibility testing for some antibiotics in 2015. A smaller dataset and high levels of data non-response for some variables limited the choice and number of risk factors included in the model, due to low numbers of individuals per stratum, which reduces the reliability of the prevalence estimates used. For example, the site of sample origin (such as urethral, cervical or pharyngeal) was not included in the model due to a large amount of missing data for this variable. This may limit the model as infections are known to be more likely to harbour resistance; they can also be more difficult to treat and require ceftriaxone treatment regardless. ${ }^{4728}$ Similarly, because only a limited number of variables could be included in the model, this simple approach predicted individual susceptibility based on broad individual categories, which may not be the most acceptable or accurate approach. More broadly, re-introducing old antibiotics could increase the selection pressure for AMR, even if the study aimed to re-introduce these antibiotics conservatively. The simulation may not fully represent the real impact of the tool due to assumptions such as no loss-to-follow-up and no contraindications for any antibiotics. ${ }^{29}$

\section{Strengths and weaknesses in relation to other studies}

To enable individualised treatment, other studies have focused on biochemical techniques, such as nucleic acid amplification tests (NAATs), rather than mathematical modelling. ${ }^{6}$ Rapid NAAT point-of-care tests are being developed to quickly determine the presence of $N$. gonorrhoeae in clinical isolates and its susceptibility to multiple antibiotics, based on the presence or absence of molecular markers associated with resistance. This could facilitate rapid individualised treatment by providing results within 90 min. $^{70-33}$ For example, molecular assays can accurately determine resistance to ciprofloxacin by detecting alterations at the gonococcal GyrA 591 locus, which account for most ciprofloxacin resistance. ${ }^{34}{ }^{35}$ The modelling approach might be less acceptable than biochemical techniques as it relies on population-level assumptions about individual resistance, rather than using specimens from the individuals themselves. However, mathematical modelling could be cheaper, less technically difficult and more applicable worldwide than biochemical techniques as it does not require specialised equipment, only a simple Excel model which provides information instantaneously, provided that local GRASP-like surveillance data are available. ${ }^{36}$ Other molecular approaches include the use of whole genome sequencing (WGS). Predictive modelling and machine learning algorithms have been developed to predict resistance phenotype from the genetic sequence of $N$. gonorrhoeae. ${ }^{33} 3738$ While this approach incorporates more complex and diverse mechanisms of resistance than NAATs, it is not completely accurate at predicting individual susceptibility. ${ }^{33}{ }^{39}$ Other studies have investigated repurposing older antibiotics or those used for other diseases, such as gentamicin and aztreonam, as widespread gonococcal resistance has not emerged to these treatments. ${ }^{25} 4041$ However, they are unlikely to be recommended as first-line treatments soon due to lower efficacy or a lack of evidence for clinical efficacy. Although resistance has previously emerged to the antibiotics investigated in this study, as previous first-line treatments they are well studied and known to be effective for susceptible infections. $^{42}$

\section{Meaning of the study}

In this study, we developed a simple, user-friendly model for predicting individualised treatment for gonorrhoea, which if implemented in practice could enable use of legacy antibiotics in susceptible infections instead of last-line treatment ceftriaxone, which could prolong its effective lifetime. This is in line with UK national strategy on AMR, which aims to optimise use of antibiotics and preserve last-line therapies. ${ }^{43}$ However, we found that antibiotic resistance may not be varied enough between participant groups for heterogenous prescription of antibiotics, which is essential for the approach to be feasible. The prevalence of ciprofloxacin and penicillin resistance was too high to permit treatment, while cefixime resistance was found to be universally low across groups. However, our model recommends that almost everyone be treated with cefixime, which would likely lead to the re-emergence of resistance. Nonetheless, treating a minority of individuals with cefixime could be feasible. Azithromycin resistance was potentially varied enough between groups to facilitate heterogenous treatment; azithromycin monotherapy is known to effectively treat susceptible $N$. gonorrhoeae, so this could be a potential option for those with a low predicted risk of resistance. ${ }^{44}{ }^{45}$ However, the $2 \mathrm{~g}$ dose of azithromycin necessary to avoid the development of resistance can be associated with adverse gastrointestinal side effects. ${ }^{46}{ }^{47}$ Furthermore, this preliminary model only recommends antibiotic monotherapy, which has been associated with the spread of resistance. For example, resistance emerged to cefixime and azithromycin in Japan following the use of cefixime and azithromycin monotherapies ${ }^{58} 49$ Developing a model which can recommend individualised multi-drug therapies, with the help of clinician guidance, could help to reduce the 
risk of resistance emerging and make this approach more effective. ${ }^{741}$

This preliminary data analysis was to assess whether a tool using routine patient data to guide treatment could be useful and to stimulate further discussion in the context of growing concern about antibiotic resistance. This may also help to support decision-making around the use of new diagnostic tests to detect resistance as these become more commonly available.

\section{Unanswered questions and future research}

Future research could investigate using this approach with more robust estimates of the prevalence of resistance. This could be achieved with a larger dataset, potentially by including more years of GRASP data in the future, although using older data may make the model less reflective of the dynamic nature of changing resistance patterns in the population. Alternatively, the model could be used in conjunction with data from other surveillance systems, for example, data from the Genitourinary Medicine Clinic Activity Dataset or the Second Generation Surveillance System. ${ }^{50-52}$ The use of regional data could allow sexual networks to be included in the model and provide greater discriminatory value than patient characteristics alone. Using a larger sample size, with data from multiple sources, could result in more robust estimates of the prevalence of resistance in different participant groups. This could help to ascertain whether this approach is feasible in practice. Using a larger dataset could also enable more in-depth stratified analysis, allowing the development of alternative algorithms for determining the best risk factors to include the model, without being limited by small numbers of individuals per stratum. This could facilitate the development of a model with less broad participant categories, with greater distinguishing power, and potentially more accurate predictions for individual susceptibility. In the future, this modelling approach could also be used in conjunction with bioinformatic data from WGS to predict antibiotic susceptibility from genetic information; this aims to link genome sequence data from the gonococcal isolate to predict their phenotypic susceptibility, but is not currently completely accurate at predicting susceptibility. ${ }^{37} 3953$ Molecular assays to detect genetic markers of resistance at the patient level are another available tool which could be combined with a modelling approach. ${ }^{34}{ }^{35}$ Use of a combination of data sources in the model to inform prescribing could result in more nuanced individualised decision-making.

Importantly, future research could explore testing the model in real-life circumstances, including culture and AMR testing of samples, to determine the applicability of the model to clinics in the UK as well as the compliance of clinicians with the predictions of the model, ensuing that any change to prescribing practice was fair and optimised patient treatment. Similar user-friendly tools to predict cancer risk and antibiotic resistance risk using patient characteristics have been acceptable to clinicians provided that the tool is easy to use, quick to complete for each patient and endorsed by clinical bodies. ${ }^{26}{ }^{54-56}$ Surveillance data used in a tool to guide prescribing would also need to be accurate and relevant for the patient population. ${ }^{57}$ Further development work with clinicians could help to assess the acceptability of this prescribing approach, any barriers or facilitators to its use, and whether it could feasibly be integrated into clinical practice.

\section{Twitter Katy M E Turner @katymeturner}

Acknowledgements We acknowledge Public Health England for permitting and facilitating access to GRASP data. LF, MG and KMET acknowledge support from the NIHR Health Protection Research Unit in Behavioural Science and Evaluation at University of Bristol. KMET acknowledges support from Health Data Research UK via the Better Care Partnership Southwest (HDR CF0129).

Contributors LF contributed to the design of the study, performed data analysis and interpretation, and drafted and revised the manuscript. KMET contributed to the conception and design of the study and data analysis and interpretation. HM and MG contributed to the conception and design of the study and acquisition and interpretation of data. JR and HF contributed to the conception and design of the study and interpretation of data. OGO contributed towards acquisition and interpretation of data. All authors revised the manuscript critically and approved the final version.

Funding The authors have not declared a specific grant for this research from any funding agency in the public, commercial or not-for-profit sectors.

Disclaimer The views expressed are those of the author and not necessarily those of the NIHR, the Department of Health and Social Care, or PHE.

Competing interests JR reports personal fees from GSK Pharma, Mycovia and Nabriva Therapeutics as well as ownership of shares in GSK Pharma and AstraZeneca Pharma; and is author of the UK and European Guidelines on Pelvic Inflammatory Disease; is a Member of the European Sexually Transmitted Infections Guidelines Editorial Board; is a Member of the National Institute for Health Research Funding Committee (Health Technology Assessment programme). $\mathrm{He}$ is an NIHR Journals Editor and associate editor of Sexually Transmitted Infections journal. He is an officer of the International Union against Sexually Transmitted Infections (treasurer) and a charity trustee of the Sexually Transmitted Infections Research Foundation. KMET has received grant funding from GlaxoSmithKline and consultancy fees from Aquarius Population Health for work outside this project.

Patient consent for publication Not required.

Ethics approval Permission was obtained to access GRASP surveillance data stored and collected by Public Health England (PHE), under the Data Protection Act (1998) and the STI and HIV Data Sharing Policy. All individual-level data extracted from GRASP were pseudonymised and accessed from a secure PHE laptop and server and were not taken from PHE premises. The GRASP data were used to estimate model parameters which were not considered confidential (for example, the prevalence of antibiotic resistance). Ethical approval for the study was obtained from the University of Bristol Ethics Committee (ID: 87902).

Provenance and peer review Not commissioned; externally peer reviewed.

Data availability statement Data are available on reasonable request. The Excel spreadsheet and user guide are available as supplemental materials. The GRASP dataset used is available on request from Public Health England.

Supplemental material This content has been supplied by the author(s). It has not been vetted by BMJ Publishing Group Limited (BMJ) and may not have been peer-reviewed. Any opinions or recommendations discussed are solely those of the author(s) and are not endorsed by BMJ. BMJ disclaims all liability and responsibility arising from any reliance placed on the content. Where the content includes any translated material, BMJ does not warrant the accuracy and reliability of the translations (including but not limited to local regulations, clinical guidelines, terminology, drug names and drug dosages), and is not responsible for any error and/or omissions arising from translation and adaptation or otherwise.

Open access This is an open access article distributed in accordance with the Creative Commons Attribution Non Commercial (CC BY-NC 4.0) license, which permits others to distribute, remix, adapt, build upon this work non-commercially, and license their derivative works on different terms, provided the original work is 
properly cited, appropriate credit is given, any changes made indicated, and the use is non-commercial. See: http://creativecommons.org/licenses/by-nc/4.0/.

\section{ORCID iDs}

Lucy Findlater http://orcid.org/0000-0003-1736-7661

Helen Fifer http://orcid.org/0000-0001-7756-403X

Katy M E Turner http://orcid.org/0000-0002-8152-6017

\section{REFERENCES}

1 Public Health England. Sexually transmitted infections and screening for chlamydia in England, 2018. Public Health Engl, 2018.

2 Turner KM, Christensen H, Adams EJ, et al. Analysis of the potential for point-of-care test to enable individualised treatment of infections caused by antimicrobial-resistant and susceptible strains of Neisseria gonorrhoeae: a modelling study. BMJ Open 2017;7:e015447.

3 Goire N, Lahra MM, Chen M, et al. Molecular approaches to enhance surveillance of gonococcal antimicrobial resistance. Nat Rev Microbiol 2014;12:223-9.

4 et alFifer H, Saunders J, Soni S. BASHH guidelines: British Association for Sexual Health and HIV national guideline for the management of infection with Neisseria gonorrhoeae (2019) [Internet]. BASHH, 2019. Available: https://www.bashhguidelines.org/ media/1208/gc-2019.pdf [Accessed 17 Dec 2020].

5 Unemo M, Shafer WM. Antimicrobial resistance in Neisseria gonorrhoeae in the 21st century: past, evolution, and future. Clin Microbiol Rev 2014;27:587-613.

6 Johnson AP, Hughes G. The prospect of untreatable gonorrhoea. BMJ 2017;358:j3973.

7 Buono SA, Watson TD, Borenstein LA, et al. Stemming the tide of drug-resistant Neisseria gonorrhoeae: the need for an individualized approach to treatment. J Antimicrob Chemother 2015;70:374-81.

8 Public Health England. Antimicrobial resistance in Neisseria gonorrhoeae in England and Wales: Key findings from the Gonococcal Resistance to Antimicrobials Surveillance Programme (GRASP 2018). Data to June 2019. [Internet]. UK Government, 2019. Available: https://assets.publishing.service.gov.uk/government/ uploads/system/uploads/attachment_data/file/834924/GRASP 2018_report.pdf [Accessed 17 Dec 2020].

9 World Health Organization. WHO guidelines for the treatment of Neisseria gonorrhoeae. [Internet]. WHO, 2016. Available: http://www. ncbi.nlm.nih.gov/books/NBK379221/ [Accessed 21 Aug 2019].

10 Centres for Disease Control and Prevention (CDC). Gonococcal Infections - 2015 STD Treatment Guidelines [Internet], 2019. Available: https://www.cdc.gov/std/tg2015/gonorrhea.htm [Accessed 21 Aug 2019].

11 Paine TC, Fenton KA, Herring A, et al. GRASP: a new national sentinel surveillance initiative for monitoring gonococcal antimicrobial resistance in England and Wales. Sex Transm Infect 2001;77:398-401.

12 Town K, Obi C, Quaye N, et al. Drifting towards ceftriaxone treatment failure in gonorrhoea: risk factor analysis of data from the Gonococcal Resistance to Antimicrobials Surveillance Programme in England and Wales. Sex Transm Infect 2017;93:39-45.

13 Andrews JM. Determination of minimum inhibitory concentrations. $J$ Antimicrob Chemother 2001;48 Suppl 1:5-16.

14 Sánchez-Busó L, Golparian D, Corander J, et al. The impact of antimicrobials on gonococcal evolution. Nat Microbiol 2019;4:1941-50.

15 Clifton S, Bolt H, Mohammed H, et al. Prevalence of and factors associated with MDR Neisseria gonorrhoeae in England and Wales between 2004 and 2015: analysis of annual cross-sectional surveillance surveys. J Antimicrob Chemother 2018;73:923-32.

16 Public Health England. Surveillance of antimicrobial resistance in Neisseria gonorrhoeae in England and Wales. Key findings from the Gonococcal Resistance to Antimicrobials Surveillance Programme (GRASP). Data to May 2018. [Internet]. UK Government, 2018. Available: https://assets.publishing.service.gov.uk/government/ uploads/system/uploads/attachment_data/file/746261/GRASP 2017 report.pdf [Accessed 17 Dec 2020].

17 Public Health England. Surveillance of antimicrobial resistance in Neisseria gonorrhoeae: Key findings from the Gonococcal Resistance to Antimicrobials Surveillance Programme (GRASP). Data up to October 2016. [Internet]. UK Government, 2016. Available: https://assets.publishing.service.gov.uk/government/uploads/ system/uploads/attachment_data/file/771134/GRASP_Report_2016. pdf [Accessed 17 Dec 2020].

18 The European Committee on Antimicrobial Susceptibility Testing. Breakpoint tables for interpretation of MICs and zone diameters.
Version 8.1. [Internet], 2018. Available: http://www.eucast.org [Accessed 24 Mar 2021]

19 The European Committee on Antimicrobial Susceptibility Testing. Breakpoint tables for interpretation of MICs and zone diameters. Version 11.0 [Internet], 2021. Available: https://www.eucast.org [Accessed 24 Mar 2021]

20 Public Health England. Antimicrobial resistance in Neisseria gonorrhoeae in England and Wales: key findings from the Gonococcal Resistance to Antimicrobials Surveillance Programme (GRASP 2019). Data to June 2020 [Internet]. UK Government, 2020. Available: https://assets.publishing.service.gov.uk/government/ uploads/system/uploads/attachment_data/file/936166/GRASP 2019_report.pdf [Accessed 25 Mar 2021]

21 de Vries HJC, van der Helm JJ, Schim van der Loeff MF, et al Multidrug-resistant Neisseria gonorrhoeae with reduced cefotaxime susceptibility is increasingly common in men who have sex with men, Amsterdam, the Netherlands. Euro Surveill 2009;14:19330.

22 Brooks H, Llewellyn CD, Nadarzynski T, et al. Sexual orientation disclosure in health care: a systematic review. Br J Gen Pract 2018;68:e187-96.

23 World Health Organization. Guidelines for the management of sexually transmitted infections. [Internet]. World Health Organization, 2003. Available: https://apps.who.int/iris/bitstream/ handle/10665/42782/9241546263_eng.pdf;jsessionid=BECB6311 C3D6A01119EAB0FA4B543DD4? sequence $=1$ [Accessed 17 Dec 2020].

24 Turner KME, Hutchinson S, Vickerman P, et al. The impact of needle and syringe provision and opiate substitution therapy on the incidence of hepatitis $C$ virus in injecting drug users: pooling of UK evidence. Addiction 2011;106:1978-88.

25 Ross JDC, Lewis DA. Cephalosporin resistant Neisseria gonorrhoeae: time to consider gentamicin? Sex Transm Infect 2012;88:6-8.

26 Yelin I, Snitser O, Novich G, et al. Personal clinical history predicts antibiotic resistance of urinary tract infections. Nat Med 2019;25:1143-52.

27 Weinstock H, Workowski KA. Pharyngeal gonorrhea: an important reservoir of infection? Clin Infect Dis 2009;49:1798-800.

28 Regan DG, Hui BB, Wood JG, et al. Treatment for pharyngeal gonorrhoea under threat. Lancet Infect Dis 2018;18:1175-7.

29 National Institute for Health and Care Excellence. BNF (British National Formulary): Cefixime [Internet]. Available: https://bnf.nice. org.uk/drug/cefixime.html\#indicationsAndDoses [Accessed 24 Aug 2019].

30 Gaydos CA, Van Der Pol B, Jett-Goheen M, et al. Performance of the Cepheid CT/NG Xpert rapid PCR test for detection of Chlamydia trachomatis and Neisseria gonorrhoeae. J Clin Microbiol 2013;51:1666-72.

31 Adams EJ, Ehrlich A, Turner KME, et al. Mapping patient pathways and estimating resource use for point of care versus standard testing and treatment of chlamydia and gonorrhoea in genitourinary medicine clinics in the UK. BMJ Open 2014;4:e005322.

32 Fingerhuth SM, Low N, Bonhoeffer S, et al. Detection of antibiotic resistance is essential for gonorrhoea point-of-care testing: a mathematical modelling study. BMC Med 2017;15:142.

33 Hicks AL, Wheeler N, Sánchez-Busó L, et al. Evaluation of parameters affecting performance and reliability of machine learning-based antibiotic susceptibility testing from whole genome sequencing data. PLoS Comput Biol 2019;15:e1007349.

34 Trembizki E, Guy R, Donovan B, et al. Further evidence to support the individualised treatment of gonorrhoea with ciprofloxacin. Lancet Infect Dis 2016;16:1005-6.

35 Ebeyan S, Windsor M, Bordin A, et al. Evaluation of the ResistancePlus GC (beta) assay: a commercial diagnostic test for the direct detection of ciprofloxacin susceptibility or resistance in Neisseria gonorrhoeae. J Antimicrob Chemother 2019;74:1820-4.

36 Bissessor M, Tabrizi SN, Fairley CK, et al. Differing Neisseria gonorrhoeae bacterial loads in the pharynx and rectum in men who have sex with men: implications for gonococcal detection, transmission, and control. J Clin Microbiol 2011;49:4304-6.

37 Eyre DW, De Silva D, Cole K, et al. WGS to predict antibiotic MICs for Neisseria gonorrhoeae. J Antimicrob Chemother 2017;72:1937-47.

38 Břinda K, Callendrello A, KC M. Rapid heuristic inference of antibiotic resistance and susceptibility by genomic neighbor typing. bioRxiv 2019 https://www.biorxiv.org/content/

39 Mortimer TD, Grad YH. Applications of genomics to slow the spread of multidrug-resistant Neisseria gonorrhoeae. Ann N Y Acad Sci 2019;1435:93-109.

40 Davido B, Dinh A, Senard O, et al. Repurposing an old drug: aztreonam as a new treatment strategy for gonorrhoea. J Antimicrob Chemother 2017;72:dkw589-8. 
41 Suay-García B, Pérez-Gracia MT. Future prospects for Neisseria gonorrhoeae treatment. Antibiotics 2018;7:49.

42 Ross JDC, Brittain C, Cole M, et al. Gentamicin compared with ceftriaxone for the treatment of gonorrhoea (G-ToG): a randomised non-inferiority trial. Lancet 2019;393:2511-20.

43 HM Government. Tackling antimicrobial resistance 2019-2024: The UK's five-year national action plan. HM Government, 2019.

44 Katz KA, Pierce EF, Aiem H. Neisseria gonorrhoeae with reduced susceptibility to azithromycin. Medscape Morb Mortal Wkly Rep 2011:60:579-81.

45 National Institute for Health and Care Excellence. BNF (British National Formulary): Azithromycin [Internet]. Available: https://bnf. nice.org.uk/drug/azithromycin.html [Accessed 25 Aug 2019].

46 Yasuda $\mathrm{M}$, Ito $\mathrm{S}$, Kido $\mathrm{A}$, et al. A single $2 \mathrm{~g}$ oral dose of extendedrelease azithromycin for treatment of gonococcal urethritis. $J$ Antimicrob Chemother 2014;69:3116-8.

47 Soda M, Ito S, Matsumaru N, et al. Evaluation of the microbiological efficacy of a single 2-gram dose of extended-release azithromycin by population pharmacokinetics and simulation in Japanese patients with gonococcal urethritis. Antimicrob Agents Chemother 2018:62:e01409-17.

48 Tanaka M, Nakayama $\mathrm{H}$, Tunoe $\mathrm{H}$, et al. A remarkable reduction in the susceptibility of Neisseria gonorrhoeae isolates to cephems and the selection of antibiotic regimens for the single-dose treatment of gonococcal infection in Japan. J Infect Chemother 2002;8:81-6.

49 Morita-Ishihara T, Unemo M, Furubayashi K-I, et al. Treatment failure with $2 \mathrm{~g}$ of azithromycin (extended-release formulation) in gonorrhoea in Japan caused by the international multidrug-resistant ST1407 strain of Neisseria gonorrhoeae. J Antimicrob Chemother 2014;69:2086-90.
50 Savage EJ, Mohammed $\mathrm{H}$, Leong G, et al. Improving surveillance of sexually transmitted infections using mandatory electronic clinical reporting: the genitourinary medicine clinic activity dataset, England, 2009 to 2013. Euro Surveill 2014;19:20981.

51 Public Health England. GUMCAD STI surveillance system: data specification and technical guidance. Public Health England, 2019.

52 Public Health England. Laboratory reporting to public health England: a guide for diagnostic libraries. Public Health England, 2016.

53 Cristillo AD, Bristow CC, Torrone E, et al. Antimicrobial resistance in Neisseria gonorrhoeae: proceedings of the StAR sexually transmitted infection-clinical trial group programmatic meeting. Sex Transm Dis 2019;46:e18-25.

54 Archer S, Babb de Villiers C, Scheibl F, et al. Evaluating clinician acceptability of the prototype CanRisk tool for predicting risk of breast and ovarian cancer: a multi-methods study. PLoS One 2020;15:e0229999.

55 Lodise TP, Bonine NG, Ye JM, et al. Development of a bedside tool to predict the probability of drug-resistant pathogens among hospitalized adult patients with gram-negative infections. BMC Infect Dis 2019;19:718.

56 Public Health England. Behaviour change and antibiotic prescribing in healthcare settings: literature review and behavioural analysis [Internet], 2015. Available: https://assets.publishing.service.gov. uk/government/uploads/system/uploads/attachment_data/file/ 774129/Behaviour_Change_for_Antibiotic_Prescribing_-_FINAL.pdf [Accessed 21 Dec 2020].

57 Anderson EC, Kesten JM, Lane I, et al. Primary care clinicians' views of paediatric respiratory infection surveillance information to inform clinical decision-making: a qualitative study. BMJ Paediatr Open 2019;3:e000418. 